\title{
Evaluation of Perinatal Mortality Surveillance System in the City of Bulawayo, Zimbabwe
}

\section{Munekayi Padingani1 ${ }^{*}$, Gladys Marape ${ }^{2}$, Zanele Hwalima ${ }^{2}$, Lucia Takundwa ${ }^{1}$, Notion Gombe ${ }^{3}$, Gerald Shambira1, Tsitsi Juru1, Mufuta Tshimanga1}

\author{
${ }^{1}$ Department of Community Medicine, University of Zimbabwe, Harare, Zimbabwe \\ ${ }^{2}$ City Health Department, Bulawayo City Councils, Bulawayo, Zimbabwe \\ ${ }^{3}$ African Field Epidemiology Network, Kampala, Uganda \\ Email: ^drmunepadi@gmail.com, tsitsijuru@gmail.com, marapeg78@gmail.com,zanelehwalima@gmail.com, \\ ltakundwa@gmail.com,gombent@gmail.com,gshambira@yahoo.com, tshimangamufuta@gmail.com
}

How to cite this paper: Padingani, M. Marape, G., Hwalima, Z., Takundwa, L., Gombe, N., Shambira, G., Juru, T. and Tshimanga, M. (2021) Evaluation of Perinatal Mortality Surveillance System in the City of Bulawayo, Zimbabwe. Open Journal of Epidemiology, 11, 124-134.

https://doi.org/10.4236/ojepi.2021.112012

Received: December 28, 2020

Accepted: April 19, 2021

Published: April 22, 2021

Copyright $\odot 2021$ by author(s) and Scientific Research Publishing Inc. This work is licensed under the Creative Commons Attribution International License (CC BY 4.0).

http://creativecommons.org/licenses/by/4.0/

\begin{abstract}
Background: In Zimbabwe, the perinatal mortality surveillance system is based on passive reporting of perinatal deaths using the perinatal death notification forms. Mpilo hospital recorded 74 perinatal deaths from January to September. No death was reported to the city and no perinatal mortality forms were found at the health information section. We aimed to assess the performance of perinatal mortality surveillance system in Bulawayo city. Methods: We conducted a descriptive cross-sectional study in all the maternity centres in Bulawayo City in 2011 using Centers for Disease Control and Prevention guidelines for evaluating public health surveillance systems. We used interviewer-administrated questionnaires to collect data. We used a checklist to assess availability of resources. Perinatal death notification forms were reviewed. Results: We interviewed 67 workers. Knowledge on the system was poor. Eight (12\%) were trained in IDSR (Integrated Disease Surveillance Response) and three (4.5\%) were inducted on the perinatal mortality surveillance system. Sixty-one (91\%) regarded the system as useful. City and private maternity centres were not notifying perinatal deaths due to lack of forms. In central hospitals, delay in notification was due to workload and shortage of trained staff. Conclusion: Poor knowledge on the system, lack of induction and trained staff could have contributed to the non-performance of the system in the City of Bulawayo. Most participants reported the system as useful. Lack of documentation made it difficult to follow up on actions plans.
\end{abstract}

\section{Keywords}

Evaluation, Perinatal Mortality, Surveillance System, Maternity Centres, Bulawayo City, Zimbabwe 


\section{Introduction}

World Health Organization (WHO) defines perinatal mortality as deaths occurring during late pregnancy ( $>22$ weeks of gestation), during birth and within seven days after delivery [1].

Perinatal mortality rate is defined as number of stillbirths and deaths in the first week of life per 1000 live births [2]. Perinatal mortality surveillance system is one of the many surveillance systems in Zimbabwe. It was introduced in the country in order to assess maternal health, to know the causes of perinatal death and to reach the Millennium Development Goals; Goal 4: reduce child mortality.

A mortality audit is the process of capturing information on the number and causes of deaths whether for maternal deaths, stillbirths, or neonatal deaths and identifying specific cases for systematic, critical analysis of underlying demandand supply-side contributors, including quality of care received, in a no-blame, interdisciplinary setting to improve the care provided to all mothers and babies [3]. Audits help examine the circumstances surrounding each death, including any breakdowns in care from the household to the health facility that may have been preventable. This process is an important continuous action cycle for quality improvement that can link data from the local to the national level [3].

In Zimbabwe, the perinatal mortality surveillance system is based on passive reporting of perinatal death using the perinatal death notification forms (called T8).

In case of perinatal death, the diagnosing health worker makes a verbal report first to the next level using the fastest means possible, which is then followed by a written notification using the perinatal death notification forms. The forms are completed in triplicate within 7 days of a perinatal death; the original is kept at the institution where the death occurred while the other two copies are sent to the District Medical Officer (DMO) at district level who in turn submits the copies to the Provincial Medical Director (PMD) or to the Clinical Director in case of central hospitals or to the Director of Health Services in case of city clinics and private institutions in the city within 28 days of the perinatal death. The Provincial Medical Director (PMD) or Clinical Director or Director of Health Services will complete the relevant sections on both forms and submit one copy to Ministry of Health and Child Care Reproductive Health Unit within 60 days of the perinatal death. An effective disease surveillance system should provide good quality data that can be used for planning, decision making and resource allocation [4].

The objectives of the perinatal death notification form are: to assess maternal health, to monitor the accessibility to health services and the quality of care in health facilities, to identify risk factors and high risk areas for perinatal mortality to inform programme decision-makers, to determine common causes of perinatal deaths, to estimate the burden and the workload of health facilities, to estimate and monitor perinatal mortality rates and evaluate programmes aimed at reducing child mortality. 
Flow of information in perinatal mortality surveillance system (Figure 1) [5].

Surveillance data can guide health personnel in the decision-making needed to implement the proper strategies for disease control and prevention. Surveillance is a watchful, vigilant approach to information gathering that serves to improve or maintain the health of the population. A functional disease surveillance system is essential for defining problems and taking action. Using epidemiological methods in the service of surveillance equips district and local health teams to predict or provide early detection of outbreaks [6].

The City of Bulawayo health services providers are composed of three Central Hospitals, one Infectious hospital, 19 City Clinics among them four maternity centres, one private hospital, some private maternity centres and some uniformed forces hospitals.

Thirteen perinatal deaths were reported to the health information section during the period January to September 2011. Mpilo Maternity recorded seventy four perinatal deaths during the same period. Of these deaths none of them was reported to the city and no perinatal mortality forms were found at the health information section.

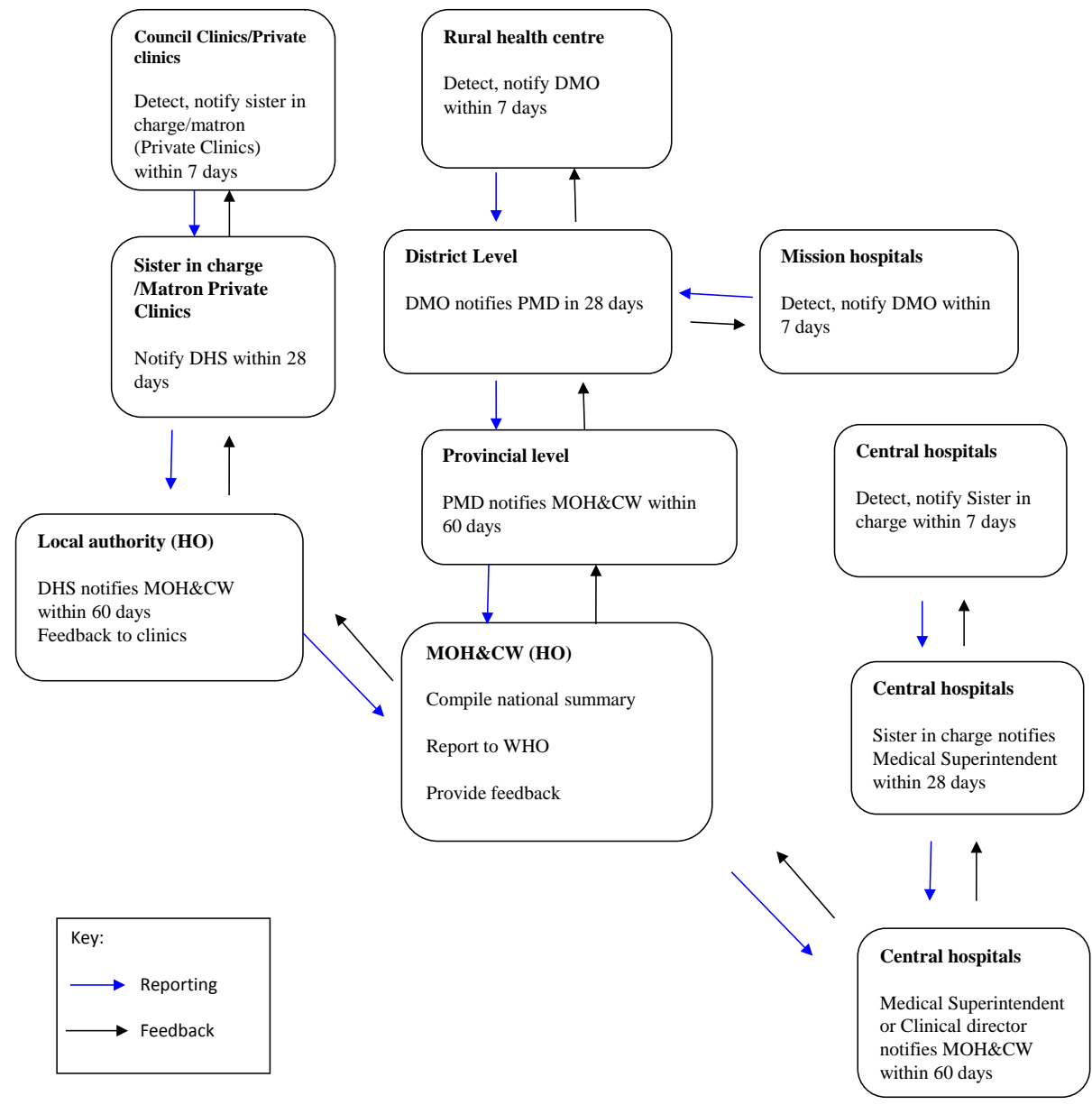

Figure 1. Flow of information in perinatal mortality surveillance system. (Adopted from Technical Guidelines for Integrated Disease Surveillance and Response in 2003.) 
Four perinatal mortality meetings are held every year in the city. Mpilo Hospital and UBH do not attend regularly yet most referrals are to these institutions. Mater Dei never attends. Reduction of child mortality is the Millennium Development Goal 4, whose target is to reduce by two-third under 5 mortality rate by 2015 [7]. Most of those deaths occur in perinatal period. The reduction of child death through effective monitoring of perinatal deaths is the goal of the national surveillance system. We therefore are willing to assess the usefulness of the system and the system's attributes.

This study assessed the performance of perinatal mortality surveillance, knowledge of health workers on perinatal surveillance, the system attributes; namely simplicity, data quality, completeness and timeliness. The acceptability, the usefulness and the stability of the system in the city of Bulawayo. Compared the performance of central hospitals, private and city clinics and identify reasons of not reporting from central and private hospitals.

\section{Methods}

A descriptive cross-sectional study was conducted in the city of Bulawayo in 2011. All health institutions offering maternity services were purposively included in the study meaning; two central hospitals, four council maternity centers, one private hospital and four private maternity centers (Mater Dei hospital, Tshabalala clinic, Marondera clinic, Pumula south clinic and Kings maternity) using Centers for Disease Control and Prevention updated guidelines for evaluating public health surveillance systems [8]. A minimum sample size of 67 participants was calculated using Dobson formula:

$$
n=Z^{2} p q / \Delta^{2} \text {. }
$$

where $n=$ sample size, $Z$ = maximum allowable error risk, $p=$ proportion of participants who felt that the perinatal mortality surveillance system is useful, $q$ $=$ proportion of health workers who did not perceive the surveillance system to be useful and $\Delta=$ absolute precision. Using a $90 \%$ confidence interval $(Z=1.64)$, an absolute a precision of $10 \%$ and we assumed $50 \%$ felt that the perinatal mortality surveillance system was useful $(P)$.

An interviewer administered questionnaire was used to collect data from key informants found on duty. Those were doctors, matrons, deputy chief nursing officer, sisters in charge, nurses, Health Information Officers from public, private hospitals and maternity city clinics to assess their knowledge on the system, gather information on the operations of the system, assess the system attributes and usefulness of the system. Knowledge of the system by health workers was referred to the understanding of the perinatal mortality surveillance. This was measured using 8 questions and marked as good if more than four (4) questions were answered correctly and poor if less than four (4). A desk review of perinatal death notification forms from October to December 2011 was conducted to assess data quality completeness, timeliness and quantity of the forms. A checklist was used to assess case definitions for perinatal death, IEC materials, IDSR 
modules, and means of communication and stocks of the perinatal death notification forms.

Permission to carry out the study was obtained from the Health Studies Office, the Director of Health Services Bulawayo City Council, Clinical directors of Central Hospitals and responsible authorities of participating private clinics and hospital.

The data was captured in Epi-Info 3.3.2 and cleaned. The same package was used to generate frequencies, proportions, tables and graphs.

Informed written consent was obtained from study participants. Confidentiality was assured and maintained throughout the study and no names were used in the questionnaire.

\section{Results}

Sixty-seven participants were interviewed. Thirty (45\%) were from Bulawayo City Council clinics, nineteen (28\%) from private institutions, eighteen (27\%) from central hospitals. The median age was 44 years (IQR: 35 - 51 Years) and median years of experience was 6.5 years (IQR: 3 - 10 Years) (Table 1). More experienced staff with more years in service was in private institutions and City clinics. Staff from central hospital had less experience.

\subsection{Knowledge of Participants on Perinatal Mortality Surveillance System}

Forty-eight (72\%) health workers correctly stated the definition of surveillance, sixteen (24\%) knew the definition of perinatal death, forty (60\%) correctly stated the two main objectives of perinatal mortality surveillance system and twenty two (33\%) knew how many perinatal death notification forms to fill in case of perinatal death. Thirty five (52\%) participants knew where the completed forms were sent to, twenty five $(37 \%)$ knew how the forms were submitted to the next level. Only nineteen (28\%) participants knew the deadline of completing the perinatal death notification forms and twenty one (31\%) knew which forms were used to report perinatal death. Out of eight questions on knowledge of the system, two were answered correctly and six were answered incorrectly by the majority of participants. On view of these answers (2/8), the knowledge of participants in perinatal mortality surveillance system was poor in the City of Bulawayo (City clinics, private institutions and central hospitals) (Table 2).

\subsection{System Attributes}

\subsubsection{Simplicity}

Four (6\%) participants only ever filled the perinatal death notification forms, all from central hospitals (Mpilo and UBH). Two out of four (50\%) perceived the system as simple and Two (50\%) described the system as difficult, citing difficulties and time consuming in filling forms.

Simplicity and flexibility of the system were not possible to be measured in City clinics and Private institutions because of unavailability of the perinatal 
death notification forms and ignorance of the perinatal mortality surveillance system by participants.

Table 1. Demographic Characteristics of Study participants.

\begin{tabular}{|c|c|c|c|c|}
\hline Respondent & $\begin{array}{l}\text { Frequency } \\
\text { (Central hospitals) }\end{array}$ & $\begin{array}{l}\text { Frequency } \\
\text { (City Clinics) }\end{array}$ & $\begin{array}{l}\text { Frequency } \\
\text { (Private institutions) }\end{array}$ & Total \\
\hline \multicolumn{5}{|l|}{ Designation } \\
\hline -Health information officer & 3 & 1 & 0 & 4 \\
\hline -Nurse & 8 & 21 & 11 & 40 \\
\hline -Sister in Charge & 2 & 4 & 4 & 10 \\
\hline -Community sister & & 4 & 0 & 4 \\
\hline -Matron & 5 & 0 & 2 & 7 \\
\hline -Doctor & 0 & 0 & 2 & 2 \\
\hline Total & 18 & 30 & 19 & 67 \\
\hline Median age in years: 44 & 31 (IQR: $35-51)$ & 44 (IQR: $41-51)$ & 49 (IQR: 45 - 55) & \\
\hline Median years of experience: 6 & 4 (IQR: 2 - 7) & 6 (IQR: 3 - 14) & 6 (IQR: 4 - 10) & \\
\hline
\end{tabular}

Table 2. Knowledge of participants on perinatal mortality surveillance system. Health Worker's knowledge on perinatal mortality surveillance system in Bulawayo, 2011.

\begin{tabular}{|c|c|c|c|c|}
\hline Variable & $\begin{array}{l}\text { Central Hospital } \\
\text { Frequency }\end{array}$ & $\begin{array}{l}\text { City clinic } \\
\text { Frequency }\end{array}$ & $\begin{array}{l}\text { Private Institution } \\
\text { Frequency }\end{array}$ & Total \\
\hline & $\mathrm{N}=18(\%)$ & $\mathrm{N}=30(\%)$ & $\mathrm{N}=19(\%)$ & $\mathrm{N}=67(\%)$ \\
\hline $\begin{array}{l}\text { Knows what } \\
\text { perinatal death is }\end{array}$ & 7 (39) & $7(23)$ & $10(53)$ & $24(36)$ \\
\hline $\begin{array}{l}\text { Knows what } \\
\text { Surveillance is }\end{array}$ & $14(78)$ & $19(63)$ & $15(79)$ & $48(72)$ \\
\hline $\begin{array}{l}\text { Knows the objectives } \\
\text { of perinatal mortality } \\
\text { surveillance system }\end{array}$ & $13(72)$ & $14(47)$ & $13(68)$ & $40(60)$ \\
\hline $\begin{array}{l}\text { Knows which form used } \\
\text { to report perinatal death }\end{array}$ & $4(22)$ & $10(33)$ & $7(37)$ & $21(31)$ \\
\hline $\begin{array}{l}\text { Knows how many } \\
\text { perinatal death } \\
\text { notification forms } \\
\text { are filled }\end{array}$ & $8(44)$ & $6(20)$ & $8(42)$ & $22(33)$ \\
\hline $\begin{array}{l}\text { Knows deadline for } \\
\text { completing the } \\
\text { perinatal death } \\
\text { notification form }\end{array}$ & 7 (39) & $7(23)$ & $5(26)$ & $19(28)$ \\
\hline $\begin{array}{l}\text { Knows where the } \\
\text { completed forms } \\
\text { are sent to }\end{array}$ & $8(44)$ & $17(57)$ & $10(53)$ & $35(52)$ \\
\hline $\begin{array}{l}\text { Knows how the forms } \\
\text { are submitted to } \\
\text { the next level }\end{array}$ & $9(50)$ & $12(40)$ & $4(21)$ & $25(37)$ \\
\hline
\end{tabular}




\subsubsection{Data Quality}

All participants did not know if the forms were checked by their supervisors before submission to next level. The Health information got reports from every maternity centres including Mpilo Central Hospital and United Bulawayo Hospitals through T5 but late after many months of delay. Seventy eight forms were reviewed. From those nine (11\%) were complete and sixty nine (89\%) incomplete (there were blank spaces).

\subsubsection{Timeliness and Completeness}

Fifty five (82\%) respondents knew that there was a deadline for submitting perinatal death notification forms to Bulawayo City Council Health Information Section then to Ministry of Health and Child Care reproductive department or straight to Ministry. In central hospitals where the perinatal death notification forms were filled, UBH missed the deadline at least seven times in the last three months of 2011 but Mpilo hospital has never met the deadline during the same period. A total of 290 perinatal deaths were reported during the period under review (October to December 2011). However, only 78 forms were submitted to the Ministry of Health and Child Care. All the 78 (100\%) forms were submitted to the Ministry within 60 days of occurrence of perinatal deaths with delay in notification which was done after 7 days. 212 perinatal deaths were not notified to the Ministry. Sixty nine (89\%) forms were incomplete with sections not filled.

Reasons cited for missing deadline were trained staff shortage and workload.

Reason for not notifying was lack of perinatal death notification forms.

\subsection{Acceptability of Perinatal Mortality Surveillance System}

Fifty five participants (82\%) were willing to participate in the perinatal mortality surveillance system. Seven (10\%) participants felt that sister in charge should take up the responsibility of filling the forms. Twenty four (36\%) participants felt that the diagnosing health worker/clinician should fill the forms. The participants who ever completed the forms felt that the completion of the perinatal death notification forms was time consuming.

Private Institutions and City clinics used still birth notification form instead of perinatal death notification form to notify perinatal death so early neonatal deaths were not notified. Those still birth notification forms were not submitted to the Health Information Section Office of City Council of Bulawayo Health Services Department.

\subsection{Usefulness of Perinatal Mortality Surveillance System}

Sixty one (91\%) participants reported that the perinatal mortality surveillance system was useful. Forty six (69\%) reported using information generated by the perinatal mortality surveillance system at local level. Only United Bulawayo Hospitals, City Clinics and Mater Dei were able to show evidence of the use of data in form of minutes of meetings. 


\subsection{Stability of Perinatal Mortality Surveillance System}

Health workers' median number years in services was 6 years $(\mathrm{Q} 1=3, \mathrm{Q} 3=10)$. Eight (12\%) of respondents were trained in IDSR while three (4\%) only from central hospitals were inducted in perinatal mortality surveillance system. Of those who did not have induction, one was responsible for filling the perinatal death notification forms.

Perinatal death notification forms were available in central hospitals and $\mathrm{Bu}$ lawayo city council health department but not available in Bulawayo City council clinics and private institutions. All the centres had functional telephones. Central hospital, Bulawayo City Council and Mater dei had computer, printing facility and a working transport system. No transport system, no computer and no printing facility for the rest of private institutions which include Kings Polyclinic, Marondera polyclinic, Pumula south clinic and Tshabalala clinic. The last two liaised with City Council transport system because they were using Council premises. All centres did not have case definition of perinatal death.

\section{Discussion}

Knowledge on the perinatal mortality surveillance system was poor among health workers. Lack of knowledge of the system by health workers could have contributed to the non-performance of the system in the City of Bulawayo since health workers did not know the definition of perinatal death such that even if perinatal death occurred, they would have not been tallied as perinatal death and that could have effect the sensitivity of the system. This is supported by Ayat Ahmadi, who found that physicians in larger cities were generally not aware of what they needed to report. Even if they did, they did not know where they should report to [9]. M. Muchekeza, found that lack of knowledge and lack of training on surveillance system for health workers were part the challenges of the system [10].

The majority (91\%) of participants reported the system as useful. Level of usefulness has to be described by actions taken as a result of the data from the surveillance system [11]. However the poor documentation at facility level made it difficult to objectively evaluate the use of the data at this level. The absence of documentation made it difficult to follow up on actions plans. The objective of a surveillance system is to produce information for action and hence it is critical to document results of analysis and recommendations including the person responsible so that individuals feel responsible and accountable. If this is not documented it is easy to forget, rendering the surveillance system useless.

None of the centres were receiving feedback from the head office. Feedback is an important part of the surveillance system loop. Giving feedback to lower level in surveillance makes health workers feel useful, knowing that they spend time doing something that is important and appreciated. Failure to give feedback may lead to demotivation which may impact negatively on the data quality.

United Bulawayo Hospitals, City Clinics and Mater Dei were receiving feed- 
back in form of perinatal and maternal mortality meetings at local level. This contrasts with results in Mutare district, 2003, where Kangwende R.A, et al, reported there was no feedback at all [12].

All the four health workers who had ever filled the forms indicated that filling in perinatal death notification forms was difficult and time consuming. Those health workers indicated that special training and good collaboration (team spirit) between health workers were required for them to be able to fill in the forms correctly.

The perception by health workers that the system was not simple may have a negative effect on the system if they are afraid to start the notification process. Few participants were trained in IDSR as well as inducted in the perinatal mortality surveillance system. This may have a negative impact on the surveillance system as training was important in giving them confidence, responsibility and the necessary skills in notifying and investigating notifiable diseases. Similar findings were noted by Sibanda C et al. (2010) in Tsholotsho district, Matebeleland North Province, on T1 surveillance (Dog bite notifications), where delay and non notification of dog bites was a result of health workers not trained in Integrated Disease Surveillance and Response and shortage of T1 forms [13].

Fifty five $(82 \%)$ health workers were willing to participate in the system. However they indicated that they would perform better if they got training in the perinatal mortality surveillance system. The willingness of health workers to take part in the surveillance system is very important for the proper performance of the system and could ease the resuscitation and strengthening of the system in the city. This finding is also supported by the results of a study conducted in Sanyati by Maponga et al. (2011) were acceptability was 100\% [14].

System stability was affected by shortage of trained staff in perinatal mortality surveillance system. Another problem which affected stability of the system was unavailability of perinatal death notification forms at City clinics and private institutions and lack of case definition on perinatal death at all institutions.

\section{Conclusions}

The perinatal mortality surveillance system in Bulawayo City was useful, acceptable, flexible but not simple, not representative, not sensitive, not stable. Simplicity was compromised by the quantity of information needed. Data was of good quality. However, the stability of the system was threatened by poor knowledge on the operation of the system by all institutions, lack of induction on the perinatal mortality surveillance system, few participants trained in IDSR, failure to notify by the private health institutions and city clinics due to unavailability of the perinatal death forms and lack of knowledge on the system. Perinatal deaths were reported to head office through T5 forms with notable delay due to workload.

We recommended the Ministry of Health and Child Welfare Reproductive Health Department to facilitate induction of nurses on perinatal mortality surveillance system as well as training in IDSR and add date of death on the forms; 
the Director of Health Services Department Bulawayo City Council to avail perinatal death notification forms to city clinics and private institutions; the Chief Nursing Officer to integrate perinatal mortality surveillance system in their supervisory visits to clearly communicate with the nurses on where to direct the perinatal death notifications forms and the Chief Nursing Officer and Matrons Central Hospital to make sure that perinatal death case definitions are displayed.

\section{Action taken}

Bulawayo City Council Health services department through the Director of Health distributed perinatal death notification forms to city clinics and private maternity centres. Communication between CNO (Chief Nursing Officer) and nurses has been established in order to know where to direct the perinatal death notification forms and display of perinatal death case definition was done at city clinics. The Ministry of Health through the maternal and child department simplified from three (3) pages to one (1).

What is already known on this topic?

- The highest burden of perinatal deaths is in developing countries with Africa contributing the majority.

- Perinatal death indicates poverty and weaknesses in the health care systems.

- Perinatal death notification is mandatory in Zimbabwe.

- No evidence of evaluation of perinatal mortality surveillance system in Bulawayo, Zimbabwe.

\section{What this study adds?}

- Perinatal death is notifiable in Zimbabwe, a knowledge gap regarding definition and reporting exists among health workers in different health institutions.

- There is need to simplify the form used to notify perinatal death.

- Lack of documentations makes it difficult to objectively assess the usefulness of the system.

\section{Acknowledgements}

We thank Centers for Disease Control and Prevention (CDC), Ministry of Health and Child Welfare, Health Study Office, Bulawayo City Council (BCC) and health workers who consented to participate in the study for making this research possible.

\section{Authors' Contributions}

All authors were responsible for the conception of the problem, design, collection, analysis and interpretation of data and drafting the final article. The manuscript was read and approved by all authors.

\section{Conflicts of Interest}

The authors declare no conflicts of interest regarding the publication of this paper. 


\section{References}

[1] World Health Organization (2018) Maternal and Perinatal Health. https://www.who.int/maternal child_adolescent/topics/maternal/maternal_perinata 1/en/

[2] Measure Evaluation (2018) Family Planning and Reproductive Health Indicators Database.

https://www.measureevaluation.org/prh/rh indicators/womens-health/nb/perinatal -mortality-rate-pmr

[3] Ministry of Health Zimbabwe and Partners (2017) Assessment of Maternal and Perinatal Death Surveillance and Response Implementation in Zimbabwe. Harare.

[4] Ministry of Health and Child Welfare Zimbabwe (2011) Perinatal Death Notification Form. 3.

[5] Ministry of Health and Child Welfare Zimbabwe (2002) Technical Guidelines for Integrated Disease Surveillance and Response in Zimbabwe.

[6] Ministry of Health and Child Welfare (2011) Technical Guidelines for Integrated Disease Surveillance and Response in Zimbabwe. 2nd Edition.

[7] World Health Organization (2019) We Can end Poverty Millenium Development Goals and beyond 2015. https://www.un.org/millenniumgoals/childhealth.shtml

[8] Centers for Disease Control and Prevention (2001) Updated Guidelines for Evaluating Public Health Surveillance Systems.

[9] Ahmadi, A., Nedjat, S., Gholami, J. and Majdzadeh, R. (2013) Disease Surveillance and Private Sector in the Metropolitans: A Troublesome Collaboration. International Journal of Preventive Medicine, 4, 1036-1044.

[10] Pomerai, K.W., Mudyiradima, R.F., Tshimanga, M. and Muchekeza, M. (2014) Evaluation of the Acute Flacid Paralysis (AFP) Surveillance System in Bikita District Masvingo Province 2010. BMC Research Notes, 7, Article Number: 252. https://doi.org/10.1186/1756-0500-7-252

[11] Centers for Disease Control and Prevention (1988) Guidelines for Evaluating Surveillance Systems. USA.

[12] Kangwende, R.A., Tshimanga, M.M.R. (2004) Evaluation of the T1/T2 Notification of Infectious Diseases Surveillance System in Mutare District. Manicaland Province, Zimbabwe: 2002-2003. (Unpublished)

[13] Sibanda, C. and Gombe, N.T.H.P. (2010) Evaluation of the T1 Surveillance in Tsholotsho District Matabeleland North, Zimbabwe. (Unpublished)

[14] Maponga, B.A., Chirundu, D., Shambira, G., Gombe, N.T. and Tshimanga, M. and Bangure, D. (2014) Evaluation of the Notifiable Diseases Surveillance System in Sanyati District, Zimbabwe, 2010-2011. The Pan African Medical Journal, 19, Article 278. https://doi.org/10.11604/pamj.2014.19.278.5202 